\title{
A comparative study of the performance of some density functionals for vibronic spectra ${ }^{\dagger}$
}

\author{
CH SRIDHAR REDDY ${ }^{\mathrm{a}, \mathrm{b}}$ and M DURGA PRASAD ${ }^{\mathrm{a}, * \mathbb{B}}$ \\ ${ }^{a}$ School of Chemistry, University of Hyderabad, Hyderabad, Telangana 500 046, India \\ ${ }^{\mathrm{b}}$ Department of Chemistry, Rajiv Gandhi University of Knowledge and Technologies, Basar, Telangana \\ 504 107, India \\ E-mail: mdpsc@uohyd.ac.in
}

MS received 30 January 2017; revised 9 April 2017; accepted 12 April 2017

\begin{abstract}
Computed vibronic spectra of four molecules, tetracene, octatetraene, anthracene and pyrene are compared to the experimental spectra with a view to determine the functional that can give the best description. Using a statistical analysis, it is found that M06-2X is best suited for reproducing the 0-0 transition energy while PBE0 and M06 are the best suited functionals for predicting the vibrational frequencies. All the functionals perform equally well in predicting the intensities of vibronic transitions.
\end{abstract}

Keywords. Electronic absorption; finite temperature; vibronic spectra; density functionals; Gaussian wave packet propagation.

\section{Introduction}

Electronic excitation of a molecule is always accompanied by vibrational excitations due to the differences in the equilibrium structures and the hessian matrices in the two electronic states. Such vibronic fine structure contains valuable information regarding the vibrational frequencies and excited state geometry. The proper interpretation of the absorption band can give a good indication of the photophysics of the molecule under consideration.

Calculation of the absorption band consists of two steps and several approximations. In the first step, one obtains the electronic potential energy surfaces of the two electronic states involved in the transition. In the second step one obtains the vibrational eigenstates of the two surfaces. The dipole matrix elements between these two sets of states are evaluated from which the spectrum is constructed. Separation of the calculation of the vibronic spectra into these parts is a consequence of the Born-Oppenheimer approximation. Even with this, the computation is still a formidable task. To simplify the procedure such that practical computations can be carried out on medium to large scale molecules, additional approximations are made. The two potential

\footnotetext{
*For correspondence

$\dagger$ Dedicated to the memory of the late Professor Charusita Chakravarty.
}

energy surfaces are limited to quadratic terms (harmonic approximation). The dipole operator is taken to be either a constant (Franck-Condon approximation) or written as a linear combination of the displacements in normal coordinates (Hertzberg-Teller approximation). Even with these approximations the computation of vibronic spectra is a non-trivial exercise. Since the hessian matrix of the molecule changes from one electronic state to other, the normal coordinates of the two surfaces are not the same. Though the harmonic approximation gives exact solution to the two states, the evaluation of overlap between such states (or more generally the dipole matrix elements) is now a many body problem. Several approaches for the evolution of such FranckCondon factors (overlap between vibrational eigenstates of two different electronic surfaces) have been developed over the years. ${ }^{1-8}$ Several authors have described the implementation of these equations ${ }^{9-14}$ for the calculation of vibronic spectra.

To reduce the computational resources, that are required as mentioned above, several time dependent approaches have been developed over the years. ${ }^{11,15-22}$ These approaches calculate the vibronic spectrum as Fourier transform of dipole-dipole auto correlation function. They differ in the detail on the computation of the auto correlation function.

More recently, extensions of both time-independent and time-dependent methods to the calculation of spectra at finite temperature have been made. ${ }^{9,10,19,23-28}$ 
Applications of these approaches indicate that the harmonic or double harmonic approximations are capable of reproducing the experimental spectra to a good extent even for medium to large size molecules provided the underline potential energy surfaces are sufficiently accurate.

The accuracy of electronic potential energy surfaces depends on the accuracy of the electronic structure calculations. Earlier studies have used the single excitation configuration interaction (CIS) method to calculate the excited states in conjunction with Hatree-Fock ground state. ${ }^{29,30}$ More recently density functional theory (DFT) based methods have been used to generate the potential energy surfaces for vibronic calculations. ${ }^{31-35}$ These studies have shown that the DFT calculations provide a good description of the vibronic structure of the absorption/emission spectra. In addition some limited studies have been reported regarding the density functionals that provide the best description of the vibrational fine structure.

The vibrational fine structure is determined by three factors. First among them is the 0-0 transition energy, which determines the band origin. Second, the changes in the parameters that define the equilibrium structures of the ground and excited states. These changes control number of overtone transitions that are seen in the spectrum and their intensities. The third part consist of the hessian matrices which determines the vibrational frequencies that are observed in the excited state spectra and the intensity borrowing effects that arise from mode mixing (Duschinsky rotation). A functional should be able to reproduce all these factors equally well to reproduce the experimental spectra.

The goal of the present work is to asses the capability of four functionals, B3LYP, ${ }^{34}$ PBE0 (also called the PBE1PBE), ${ }^{10} \mathrm{M}^{2} 6^{35}$ and M06-2X. ${ }^{35}$ The major component of the DFT functionals is the amount of exact Hatree-Fock exchange that is included in the functionals. These four functionals contain 20, 25, 27 and 54 percent of exchange energy respectively. Thus they provide a wide range of exchange energy. As test cases we have chosen three systems, tetracene at $492 \mathrm{~K},{ }^{38}$ octatetraene at $300 \mathrm{~K}^{37}$ and octatetraene at $0 \mathrm{~K}^{36}$ to compare the computed and experimental vibronic fine structure. The vibrationally resolved experimental spectra are available and more to the point, the peaks have been assigned, and, frequency and intensity values are reported for these three molecules. Thus they provide a basis for quantitative comparison of the computed and experimental spectra.

As noted earlier, a few studies have been reported in literature that made in attempt to asses the functionals for their suitability to vibronic spectral calculations. Most of the studies made comparisons with the experimental spectra in solutions. Quantum chemical calculations for salvated molecules require an additional step to salvate the molecule explicitly. This step has its own approximations. Consequently it became difficult to separate out the errors coming from the salvation models and these that are inherent in the density functional calculations. To avoid this we have chosen the above mentioned three systems for which experimental spectra in the gas phase are available.

Yet another feature of the vibronic spectra is the band origin, i.e., the 0-0 transition energy. We have calculated this for pyrene and anthracene as well. While the experimentally resolved vibronic spectra are available for these two molecules also, the experimental frequencies and intensities were not reported. So, we used these molecules only for comparison of the $0-0$ transition energies.

\section{Theory}

We used the Gaussian Wave Packet propagation approach that was developed recently in our group for vibrationally resolved optical spectra at finite temperatures. ${ }^{24}$ we briefly review the methodology in this section. We start from the time dependent expression for molar absorptivity coefficient, $\epsilon$,

$$
\epsilon=A . \omega \cdot \int C(t) \exp (i \omega t) d t
$$

where the dipole-dipole autocorrelation function, $C$, is defined as

$$
C=T r \cdot \exp \left(-i H_{e} t\right) \exp \left(+i H_{g} t\right) \exp \left(-\beta H_{g}\right)
$$

in the Franck-Condon approximation. Here $A$ is a constant, $H_{e}$ and $H_{g}$ are the vibrational Hamiltonians of excited and ground electronic state and $\beta=\frac{1}{k_{B} T}$ is the inverse temperature. Since the time evolution operator and thermal density operator commute with each other, the equation for $C$ can be compactly written as

$$
\begin{aligned}
& C=\operatorname{Tr} \exp \left[-H\left(q, q^{\prime}\right) t\right] \rho\left(q, q^{\prime}\right) \\
& H\left(q, q^{\prime}\right)=H_{e}(q)-H_{g}\left(q^{\prime}\right) .
\end{aligned}
$$

Here $\rho\left(q, q^{\prime}\right)$ is the thermal density matrix on the ground surface.

With in the harmonic approximation frame work and using the ground state mass weighted normal coordinates as the basis for the coordinate system, the two Hamiltonians become

$$
\begin{aligned}
& H_{g}=-\frac{1}{2} \sum_{i} \frac{\partial^{2}}{\partial q_{i}^{2}}+\frac{1}{2} \sum_{i} \omega_{i}^{2} q_{i}^{2}+E_{g} \\
& H_{e}=-\frac{1}{2} \sum_{i} \frac{\partial^{2}}{\partial q_{i}^{2}}+\sum_{i} U_{i} q_{i}+\frac{1}{2} \sum_{i, j} q_{i} V_{i j} q_{j}+E_{e} .
\end{aligned}
$$


Here $E_{g}$ and $E_{e}$ are the electronic state energies at the equilibrium geometry of ground surface. $U_{i}$ and $V_{i j}$ are the gradient and hessian matrix elements of exited surface at the ground state equilibrium geometry and $\left\{\omega_{i}\right\}$ are the vibrational frequencies of ground surface.

Because the ground surface hamiltonian is separable and quadratic in all coordinates, the thermal density matrix is a multidimensional Gaussian in coordinate space.

$$
\begin{aligned}
\rho_{g}= & \prod_{i}\left\{( \frac { \omega _ { k } } { \pi } \operatorname { t a n h } ( \beta \omega _ { k } ) ) ^ { \frac { 1 } { 2 } } \operatorname { e x p } \left(\frac{-\omega_{k}}{2 \sinh \left(\beta \omega_{k}\right)}\right.\right. \\
& \left.\left.\times\left[\left(q_{k}^{2}-q_{k}^{\prime 2}\right) \cosh \left(\beta \omega_{k}\right)-2 q_{k} q_{k}^{\prime}\right]\right)\right\} .
\end{aligned}
$$

Since $H$ is also a quadratic hamiltonian, $\rho$ retains its Gaussian structure throughout its time evolution. Thus it can be written as

$$
\begin{aligned}
\rho\left(q, q^{\prime}, t\right)= & \exp \left[i \left\{\left(\mathbf{q}-\mathbf{q}^{0}\right)^{T} \mathbf{A}\left(\mathbf{q}-\mathbf{q}^{0}\right)\right.\right. \\
& \left.\left.+\mathbf{p}^{T}\left(\mathbf{q}-\mathbf{q}^{0}\right)+\gamma\right\}\right] .
\end{aligned}
$$

Here $A$ is a $2 \mathrm{~N} \times 2 \mathrm{~N}$ dimensional complex matrix. $p$ and $q^{0}$ are $2 \mathrm{~N}$ element real vectors consisting both prime and unprimed variables. $\rho\left(q, q^{\prime}, t\right)$ satisfies Schrödinger equation

$$
i \dot{\rho}\left(q, q^{\prime}, t\right)=H \rho .
$$

Substituting ansatz 6 into 7 we obtain dynamical equations for $q^{0}, p, A$ and $\gamma$ as follows ${ }^{23}$

$$
\begin{aligned}
\dot{q}_{k}^{0} & =\left(\frac{\partial H}{\partial p_{k}}\right)_{p}, \\
\dot{p}_{k} & =-\left(\frac{\partial H}{\partial q_{k}}\right)_{q^{0}}, \\
\dot{A}_{k l} & =-2 \sum_{p} A_{k p} \omega_{p} A_{p l}-\frac{1}{2}\left(\frac{\partial^{2} H}{\partial q_{k} \partial q_{l}}\right)_{q^{0}}, \\
\dot{\gamma} & =\sum_{k} \frac{P_{k} \omega_{k} P_{k}}{2}-V\left(\mathbf{q}^{0}, \mathbf{q}^{0^{\prime}}\right)+\frac{i}{2} \sum_{k} A_{k k} \omega_{k} .
\end{aligned}
$$

Note that the procedure described above is in the spirit of the Gaussian wave packet propagation technique developed by Heller. ${ }^{39}$ Once the variables defined in the ansatz 6 are determined the autocorrelation function is given by

$$
C(t)=\exp (i \boldsymbol{\Phi}) \times \sqrt{\frac{\pi^{D}}{\operatorname{det}(\mathbf{B})},}
$$

where,

$$
\begin{aligned}
\boldsymbol{\Phi} & =\mathbf{q}_{u}^{0^{\mathbf{T}}} \mathbf{A}_{p p} \mathbf{q}_{u}^{0}+i \lambda^{\mathbf{T}} \mathbf{B} \lambda+\gamma, \\
\mathbf{b} & =i\left\{2 \mathbf{q}_{u}^{0^{\mathbf{T}}}\left(\mathbf{A}_{p u}+\mathbf{A}_{p p}\right)+p_{u}^{\mathbf{T}}\right\}, \\
\mathbf{B} & =i\left\{\mathbf{A}_{u u}+\mathbf{A}_{u p}+\mathbf{A}_{p u}+\mathbf{A}_{p p}\right\}, \\
\lambda & =-\frac{1}{2} \mathbf{B}^{-1} \mathbf{b} .
\end{aligned}
$$

Once the correlation function is calculated the spectrum is obtained by Furiour transforming of it.

\section{Computational details}

The potential energy surfaces needed for the spectral calculations were obtained by four density functional methods B3LYP, PBE0, M06 and M06-2X. All these calculations were carried out with $6-311 \mathrm{G}(2 \mathrm{~d}, 2 \mathrm{p})$ basis set. There is some computational evidence that this basis set is best suited for the vibrational frequency calculation. ${ }^{40}$ All calculations were carried out with G09 suite program. ${ }^{41}$ The int=altrafine, opt=vtight were used throughout. The adiabatic hessian model ${ }^{42}$ was used to construct Potential energy surfaces. In this model the structures of the molecule in the two electronic states are optimized and the hessian and geometrical parameters are extracted for each state from Gaussian output. The normal coordinates of the ground state are constructed from the hessian of that surface. The $U$ vector and $V$ matrix were constructed from the excited surface hessian and the difference vector between the two equilibrium geometries. These were then given as input for the dynamical calculations. The time integration of the dynamical equations (Eqn 8-11) was carried out with a step length $0.02 \mathrm{fs}$ and the correlation function was sampled after every 3.62 fs for 8192 steps. This gives a frequency resolution $\delta \omega=1.12 \mathrm{~cm}^{-1}$. The correlation function was multiplied by a window function $\cos \left(\frac{\pi t}{2 T}\right)$ to avoid Gibb's phenomena and an exponentially decaying function, $\exp (-\alpha t)$, with $\alpha=22.4 \mathrm{~cm}^{-1}$ for absorbing the effects of other potential decay mechanisms.

\section{Results and Discussion}

In this section we consider the relative merits of the four functionals with respect to the calculation of 0-0 transition energies, vibrational frequencies and intensities.

\subsection{0-0 Transition energies}

The $0-0$ transition occurs at

$$
E_{0-0}=E_{F}-E_{I}+Z P E_{F}-Z P E_{I} .
$$

Here $E_{F}$ and $E_{I}$ are the electronic state energies at their respective equilibrium geometries and $Z P E_{F}$ and $Z P E_{I}$ are the zero point energies on the two surfaces. We present the data for the four molecules in Table 1. As can be seen, the M06-2X is the best choice for estimating the $0-0$ band position with PBE0 being the next best. Generally all the four functionals underestimate the $E_{0-0}$ value. It appears as if that the DFT theories 
Table 1. $0-0$ Transition energies for the four molecules in $\mathrm{cm}^{-1}$.

\begin{tabular}{lccccc}
\hline Molecule & B3LYP & PBE0 & M06 & M062X & Exp \\
\hline octatetraene & 29594 & 30223 & 29310 & 33392 & 35523 \\
anthracene & 23266 & 23951 & 23238 & 25887 & 27505 \\
pyrene & 27260 & 28058 & 27162 & 30570 & 30975 \\
tetracene & 17504 & 18075 & 17623 & 20072 & 22170
\end{tabular}

systematically underestimate the adiabatic transition energy. This observation is in line with an earlier observation of Grimme ${ }^{43}$ for BP86 and B3LYP functionals. Generally $\mathrm{E}_{0-0}$ increases with increasing contribution from the exact exchange energy (EEX). There is one exception however. The $\mathrm{E}_{0-0}$ from $\mathrm{PBE} 0$ functional with $25 \%$ EEX is in all cases higher than the $\mathrm{E}_{0-0}$ from M06 functional with $27 \% \mathrm{EEX}$, though the difference is quite small.

\subsection{Vibrational frequencies}

To understand the accuracy of vibrational frequencies we consider three spectra, that of tetracene at $492 \mathrm{~K}$, octatetraene at $300 \mathrm{~K}$ and octatetraene at $0 \mathrm{~K}$. The experimental works have reported the peak positions and intensities in these three cases. Note that the frequency of the transition is defined from the local maxima

Table 2. Computed and experimental frequencies (in $\mathrm{cm}^{-1}$ ) of tetracene at $492 \mathrm{~K}$. Experimental values adapted from ref ${ }^{38}$.

\begin{tabular}{|c|c|c|c|c|c|}
\hline S. No & B3LYP & PBE0 & M06 & M06-2X & Exp \\
\hline 1 & 310.9 & 313.1 & 310.9 & 309.8 & 300.0 \\
\hline 2 & 633.0 & 633.5 & 630.7 & 631.9 & 690.0 \\
\hline 3 & 1185.1 & 1195.2 & 1192.9 & 1150.7 & 1220.0 \\
\hline 4 & 1425.3 & 1458.9 & 1444.3 & 1437.6 & 1470.0 \\
\hline 5 & 1726.0 & 1766.4 & 1747.9 & 1721.5 & 1780.0 \\
\hline 6 & 2834.8 & 2924.6 & 2898.2 & 2870.7 & 2855.0 \\
\hline 7 & 4219.6 & 4393.0 & 4352.0 & 4282.0 & 4430.0 \\
\hline$m$ & 1.04 & 0.99 & 1.01 & 1.02 & \\
\hline$c$ & -13.840 & 15.947 & 13.084 & 10.682 & \\
\hline$\sigma$ & 48.65 & 37.39 & 36.91 & 43.16 & \\
\hline \multicolumn{3}{|c|}{$\begin{array}{l}\text { Average of experimental } \\
\text { frequencies in } \mathrm{cm}^{-1} \text { is }\end{array}$} & 1820.7 & & \\
\hline
\end{tabular}

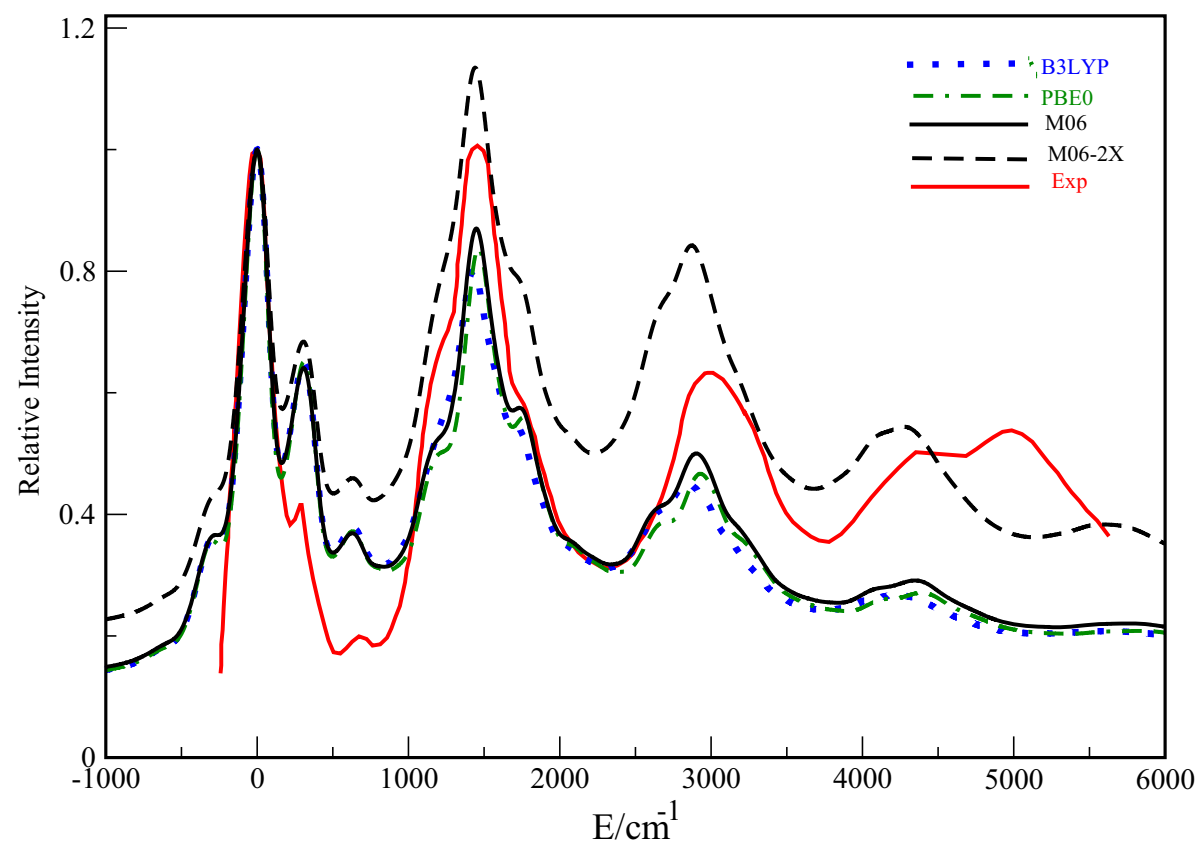

Figure 1. Comparison of calculated absorption spectrum with four functionals and experimental spectrum adapted from ref $^{38}$ at $492 \mathrm{~K}$ for tetracene. 


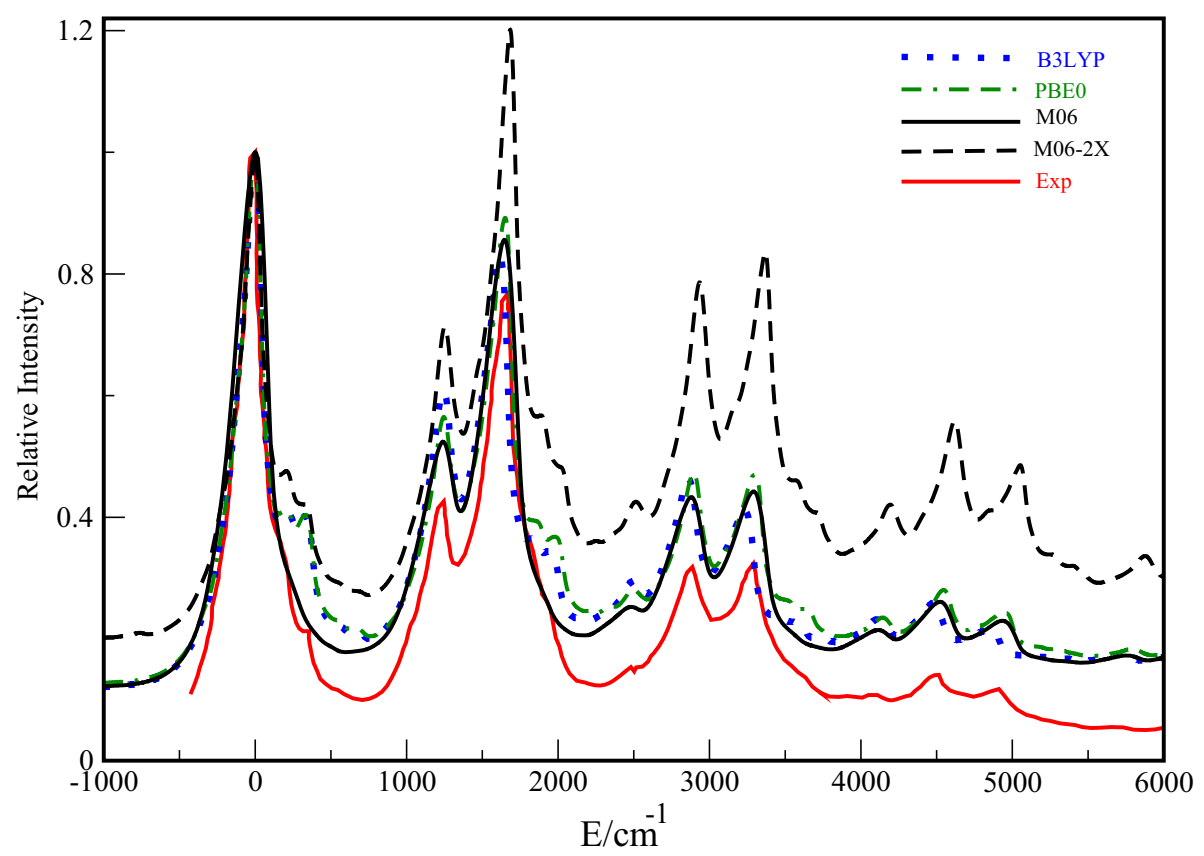

Figure 2. Comparison of calculated absorption spectrum with four functionals and experimental spectrum adapted from $\operatorname{ref}^{37}$ at $300 \mathrm{~K}$ for octatetraene.

Table 3. Computed and experimental frequencies $\left(\right.$ in $\mathrm{cm}^{-1}$ ) of octatetraene at $300 \mathrm{~K}$. Experimental values adapted from ref ${ }^{37}$.

\begin{tabular}{lrcrrr}
\hline S. No & B3LYP & PBE0 & \multicolumn{1}{c}{ M06 } & M06-2X & \multicolumn{1}{c}{ Exp } \\
\hline 1. & 218.8 & 207.6 & 205.2 & 205.3 & 199.0 \\
2. & 328.8 & 334.5 & 328.6 & 332.2 & 355.0 \\
3. & 1244.6 & 1245.7 & 1240.1 & 1252.4 & 1246.0 \\
4. & 1612.6 & 1650.8 & 1646.3 & 1684.5 & 1666.0 \\
5. & 1813.5 & 1874.7 & 1855.0 & 1885.4 & 1835.0 \\
6. & 2499.2 & 2497.0 & 2482.4 & 2513.8 & 2482.0 \\
7. & 2858.3 & 2895.4 & 2879.7 & 2934.7 & 2888.0 \\
8. & 3226.4 & 3298.3 & 3291.5 & 3367.9 & 3290.0 \\
9. & 4108.5 & 4143.3 & 4114.1 & 4193.8 & 4125.0 \\
10. & 4475.5 & 4546.2 & 4522.6 & 4619.1 & 4522.0 \\
11. & 4843.6 & 4939.0 & 4935.6 & 4854.8 & 4935.0 \\
12. & 5723.4 & 5794.1 & 5756.0 & 5877.2 & 5721.0 \\
$m$ & 1.01 & 0.99 & 0.99 & 0.98 & \\
$c$ & 7.913 & 10.071 & 12.446 & 7.014 & \\
$\sigma$ & 30.01 & 18.55 & 13.88 & 50.73 & \\
Average of experimental & 2772.0 & & \\
frequencies in $\mathrm{cm}^{-1}$ is & \multicolumn{5}{c}{} \\
\hline \multicolumn{7}{c}{}
\end{tabular}

in the spectrum. Consequently it is temperature dependent since the hotband induced line broadening need not be symmetric around the frequency of transition at $0 \mathrm{~K}$. Second, some of the transitions at $0 \mathrm{~K}$ disappear at higher temperatures due to hotband induced line broadening. it is for this reason that we have considered both $0 \mathrm{~K}$ and $300 \mathrm{~K}$ spectra of Octatetraene.

In Table 2 we present the frequencies of tetracene and compared them with the experimental frequencies. The spectrum of tetracene is presented in Figure 1. Generally all the four theoretical spectra are in good agreement with the experimental spectrum up to about $4000 \mathrm{~cm}^{-1}$ beyond the 0-0 transition. There doesn't seem to be any generic relation between EEX and frequency that we could notice. To pass a judgment on the performance of four functionals we have attempted a least square fit between the experimental and theoretical frequencies,

$$
\omega_{\text {Exp }}=m \cdot \omega_{\text {Theory }}+c .
$$

After fitting the data the standard deviation $(\sigma)$ from fitted frequencies to the experimental frequencies was 
Table 4. Computed and experimental frequencies (in $\mathrm{cm}^{-1}$ ) of octatetraene at $0 \mathrm{~K}$. Experimental values adapted from ref ${ }^{36}$.

\begin{tabular}{lccccc}
\hline S. No & B3LYP & PBE0 & \multicolumn{1}{c}{ M06 } & M06-2X & Exp \\
\hline 1 & 213.3 & 218.8 & 213.2 & 216.6 & 197.0 \\
2 & 340.1 & 341.2 & 341.1 & 343.2 & 348.0 \\
3. & 1209.8 & 1226.6 & 1218.7 & 1237.8 & 120.0 \\
4. & 1244.6 & 1251.3 & 1240.0 & 1265.9 & 1235.0 \\
5. & 1456.7 & 1460.1 & 1453.3 & 1482.5 & 1432.0 \\
6. & 1626.2 & 1655.3 & 1648.5 & 1685.6 & 1645.0 \\
7. & 1838.3 & 1864.1 & 1862.9 & 1902.2 & 1842.0 \\
8. & 1965.1 & 1996.5 & 2002.0 & 2027.9 & 1993.0 \\
9. & 2488.0 & 2502.6 & 2480.1 & 2532.9 & 2470.0 \\
10. & 2869.6 & 2906.6 & 2877.0 & 2951.5 & 2880.0 \\
11. & 3251.2 & 3310.6 & 3298.2 & 3370.1 & 3290.0 \\
12. & 3464.4 & 3519.4 & 3512.5 & 3586.6 & 3487.0 \\
13. & 3591.2 & 3651.8 & 3651.7 & 3713.5 & 3638.0 \\
14. & 4114.1 & 4157.9 & 4129.8 & 4217.3 & 4115.0 \\
15. & 4495.7 & 4561.9 & 4538.3 & 4637.1 & 4525.0 \\
16. & 4877.3 & 4965.9 & 4947.9 & 5055.7 & 4935.0 \\
$m$ & 1.01 & 0.99 & 0.99 & 0.98 & \\
$c$ & -16.090 & -9.311 & -8.098 & -4.225 & \\
$\sigma$ & 17.50 & 9.86 & 8.21 & 9.18 & \\
Average of experimental & 2452.1 & & \\
frequencies in $\mathrm{cm}^{-1}$ is & & & \\
\hline \multicolumn{7}{c}{} & & & &
\end{tabular}

Table 5. Parameters from statistical analysis including all the four molecules.

\begin{tabular}{llrlllll}
\hline \multirow{2}{*}{ Method } & \multicolumn{3}{c}{ Frequencies } & & \multicolumn{3}{c}{ Intensities } \\
\cline { 2 - 3 } \cline { 6 - 7 } & $m$ & \multicolumn{1}{c}{$c$} & $\sigma$ & & $m$ & \multicolumn{1}{c}{$c$} & $\sigma$ \\
\hline B3LYP & 1.01 & -1.622 & 39.14 & & 0.88 & -0.023 & 0.14 \\
PBE0 & 0.99 & 6.486 & 23.13 & & 0.89 & -0.015 & 0.15 \\
M06 & 1.00 & 6.966 & 24.04 & & 0.91 & 0.007 & 0.14 \\
M06-2X & 0.98 & 7.174 & 55.30 & & 0.63 & -0.042 & 0.13 \\
Average of experimental & frequencies is $2434.50 \mathrm{~cm}^{-1}$ & and intensities is 0.25
\end{tabular}

Table 6. Computed and experimental intensities of tetracene at $492 \mathrm{~K}$. Experimental values adapted from $\mathrm{ref}^{38}$.

\begin{tabular}{llllcl}
\hline S. No & B3LYP & PBE0 & M06 & M06-2X & Exp \\
\hline 1. & 0.62 & 0.62 & 0.61 & 0.65 & 0.43 \\
2. & 0.33 & 0.33 & 0.32 & 0.41 & 0.21 \\
3. & 0.49 & 0.43 & 0.45 & 0.65 & 0.83 \\
4. & 0.75 & 0.79 & 0.82 & 1.08 & 1.00 \\
5. & 0.48 & 0.50 & 0.51 & 0.71 & 0.70 \\
6. & 0.40 & 0.42 & 0.45 & 0.77 & 0.63 \\
7. & 0.23 & 0.23 & 0.25 & 0.49 & 0.51 \\
$m$ & 0.91 & 0.83 & 0.88 & 1.02 & \\
$c$ & 0.188 & 0.224 & 0.178 & -0.081 & \\
$\sigma$ & 0.19 & 0.20 & 0.19 & 0.13 & \\
\multicolumn{5}{l}{ Average of experimental } \\
intensities is
\end{tabular}

estimated. Both for tetracene data and all the three sets of data we found PBE0 has the lowest standard deviation among the four functionals with M06 being a close second.
We present in Figure 2. the spectrum of octatetraene at $300 \mathrm{~K}$ and corresponding data for frequencies are presented in Table 3. In Table 4 we present the frequencies of octatetraene both calculated and experimental at $0 \mathrm{~K}$. 
Table 7. Computed and experimental intensities of octatetraene at 300 K. Experimental values adapted from ref $^{37}$.

\begin{tabular}{lccccc}
\hline S. No & B3LYP & PBE0 & M06 & M06-2X & Exp \\
\hline 1 & 0.42 & 0.41 & 0.31 & 0.48 & 0.22 \\
2 & 0.40 & 0.40 & 0.23 & 0.35 & 0.10 \\
3 & 0.61 & 0.57 & 0.52 & 0.71 & 0.36 \\
4 & 0.83 & 0.89 & 0.86 & 1.20 & 0.63 \\
5 & 0.37 & 0.39 & 0.30 & 0.47 & 0.22 \\
6 & 0.30 & 0.28 & 0.25 & 0.43 & 0.09 \\
7 & 0.46 & 0.47 & 0.43 & 0.79 & 0.28 \\
8 & 0.41 & 0.46 & 0.44 & 0.84 & 0.32 \\
9 & 0.23 & 0.24 & 0.21 & 0.42 & 0.09 \\
10 & 0.26 & 0.28 & 0.26 & 0.56 & 0.13 \\
11 & 0.22 & 0.24 & 0.23 & 0.42 & 0.10 \\
12 & 0.18 & 0.18 & 0.17 & 0.34 & 0.06 \\
$m$ & 0.85 & 0.83 & 0.85 & 0.61 & \\
$c$ & -0.113 & -0.116 & -0.080 & -0.138 & \\
$\sigma$ & 0.05 & 0.04 & 0.02 & 0.05 & \\
Average of experimental & & 0.22 & & \\
intensities is & \multicolumn{5}{c}{} \\
\hline
\end{tabular}

Table 8. Computed and experimental intensities of octatetraene at $0 \mathrm{~K}$. Experimental values adapted from ref $^{36}$.

\begin{tabular}{lcclll}
\hline S. No & B3LYP & PBE0 & M06 & M06-2X & Exp \\
\hline 1 & 0.07 & 0.07 & 0.04 & 0.10 & 0.11 \\
2 & 0.19 & 0.17 & 0.01 & 0.12 & 0.05 \\
3 & 0.07 & 0.12 & 0.23 & 0.24 & 0.07 \\
4 & 0.34 & 0.22 & 0.11 & 0.24 & 0.23 \\
5 & 0.03 & 0.02 & 0.07 & 0.04 & 0.02 \\
6 & 0.70 & 0.77 & 0.74 & 1.08 & 0.60 \\
7 & 0.05 & 0.06 & 0.03 & 0.10 & 0.07 \\
8 & 0.12 & 0.12 & 0.01 & 0.13 & 0.05 \\
9 & 0.06 & 0.03 & 0.02 & 0.04 & 0.02 \\
10 & 0.21 & 0.17 & 0.14 & 0.30 & 0.14 \\
11 & 0.21 & 0.30 & 0.32 & 0.59 & 0.16 \\
12 & 0.02 & 0.03 & 0.01 & 0.07 & 0.03 \\
13 & 0.03 & 0.04 & 0.01 & 0.07 & 0.03 \\
14 & 0.04 & 0.02 & 0.01 & 0.05 & 0.04 \\
15 & 0.07 & 0.07 & 0.04 & 0.16 & 0.07 \\
16 & 0.05 & 0.08 & 0.08 & 0.25 & 0.06 \\
$m$ & 0.79 & 0.75 & 0.69 & 0.49 & \\
$c$ & -0.002 & 0.004 & 0.029 & 0.000 & \\
$\sigma$ & 0.04 & 0.04 & 0.06 & 0.05 & \\
Average of experimental & 0.11 & & \\
intensities is & & & & & \\
\hline
\end{tabular}

The performance of four functionals is quite similar to their performance for tetracene.

We have also carried out a statistical analysis with the data for all the observed transitions from all the molecules (in Table 5). This analysis also indicates that PBE0 and M06 are the best functionals for determining the frequencies.

\subsection{Intensities}

The intensities of the peaks from different functionals along with the experimental intensities for tetracene at $492 \mathrm{~K}$, octatetraene at $300 \mathrm{~K}$ and Octatetraene at $0 \mathrm{~K}$ are presented in Tables 6, 7 and 8 respectively. For tetracene the intensities predicted by all the density functionals 
except M06-2X underestimates the intensity. On the other hand for octatetraene all the functionals overestimate the intensities.

For a single harmonic oscillator with ground and excited states potential energy surfaces given by

$$
\begin{aligned}
V_{g} & =\frac{1}{2} \omega^{2} q^{2}, \\
V_{e} & =\frac{1}{2} \omega^{2}\left(q-q_{e}\right)^{2},
\end{aligned}
$$

the intensity of the $\mathrm{n}^{\text {th }}$ overtone is given by

$$
P_{n}=\frac{2}{n !}\left(\frac{q_{e}}{\omega}\right)^{n} .
$$

From this, and the observations regarding the intensity pattern made earlier, we conclude that the M06-2X generally overestimate the changes in the geometry during the excitation. Other three functionals however underestimate the geometry changes for tetracene and overestimate the geometry changes for octatetraene. While the volume of the data is too little to draw any meaningful conclusion, we got the impression that the four density functionals considered here overestimate the geometry changes for open chain systems, and for cyclic systems the density functionals with EEX below $30 \%$ underestimate the changes that occur during electronic excitation. These changes seem to be more or less directly related to the percentage of EEX included in the functional. However studies on the larger set of molecules is necessary to conform these results. The data of Table 5 indicates that there is not much to chose between the functionals for the estimation of intensities.

\section{Conclusions}

On the whole our studies indicate that the percentage of EEX has the direct role to play in quantitative description of the vibronic spectra. Of the four functional we considered PBE0 and M06 seem to provide the best possible description for the frequencies and intensities respectively. On the other hand M06-2X appear to be the best functional for the evaluation of 0-0 transition energy. Perhaps a hybrid calculation in which $\mathrm{E}_{0-0}$ is obtained from M06-2X and geometrical and hessian parameters are obtained either from PBE0 or M06 might provide the best overall description. Additional studies are needed to determine the relation between the nature of molecular structure and functionals we chosen to describe vibronic spectra.

\section{Acknowledgements}

Ch. Sridhar Reddy Acknowledges gratefully a sustaining fellowship from UGC and DST (JC Bose fellowship). Financial support from DST (PURSE, HPCF, FIST and IYC programs) and UGC (UPE and CAS pograms) is gratefully acknowledged.

\section{References}

1. Sharp T E and Rosenstock H M 1964 Franck-Condon Factors for Polyatomic Molecules J. Chem. Phys. 41 3453

2. Botter R, Dibeler V H, Walker J A and Rosenstock H M 1966 Experimental and Theoretical Studies of Photoionization-Efficiency Curves for $\mathrm{C}_{2} \mathrm{H}_{2}$ and $\mathrm{C}_{2} \mathrm{D}_{2}$ J. Chem. Phys. 441271

3. Doktorov E V, Malkin I A and Manko V I 1975 Dynamical Symmetry of Vibronic Transitions in Polyatomic Molecules and the Franck-Condon Principle J. Mol. Spectrosc. 561

4. Doktorov E V, Malkin I A and Manko V I 1977 Dynamical Symmetry of Vibronic Transitions in Polyatomic Molecules and the Franck-Condon Principle J. Mol. Spectrosc. 64302

5. Ruhoff $\mathrm{P}$ T 1994 Recursion Relations for MultiDimensional Franck-Condon Overlap Integrals Chem. Phys. 186355

6. Gruner D and Brumer P A 1996 Genetic Algorithm Approach to Fitting Polyatomic Spectra via Geometry Shifts Chem. Phys. Lett. 261612

7. Hazra A, Chang H H and Nooijen M 2004 First Principles Simulation of the UV Absorption Spectrum of Ethylene Using the Vertical Franck-Condon Approach J. Chem. Phys. 1212125

8. Hazra A and Nooijen M 2003 Derivation and Efficient Implementation of A Recursion Formula to Calculate Harmonic Franck-Condon Factors for Polyatomic Molecules Int. J. Quantum. Chem. 95643

9. Barone V, Bloino J, Biczysko M and Santoro F 2009 Fully Integrated Approach to Compute Vibrationally Resolved Optical Spectra: From Small Molecules to Macrosystems J. Chem. Theory Comput. 5540

10. Borrelli R, Capobianco A and Peluso A 2012 Generating Function Approach to the Calculation of Spectral Band Shapes of Free-Base Chlorin Including Duschinsky and HerzbergTeller Effects J. Phys. Chem. A 1169934

11. Avila F J, Cerezo J, Stendardo E, Improta R and Santoro F 2013 Insights for an Accurate Comparison of Computational Data to Experimental Absorption and Emission Spectra: Beyond the Vertical Transition Approximation J. Chem. Theory Comput. 92072

12. Ruhoff $\mathrm{P}$ T and Ratner M A 2000 Algorithms for Computing Franck-Condon Overlap Integrals Int. J. Quantum Chem. 77383

13. Weber J O and Hohlneicher G 2003 Franck-Condon Factors for Polyatomic Molecules Mol. Phys. 1012125

14. Callis R P, Vivian J T and Slater L S 1995 Ab Initio Calculations of Vibronic Spectra for Indole Chem. Phys. Lett. 24453 
15. Patwari G N, Wategaonkar S and Prasad M D 2001 Franck-Condon Spectral Calculations on TransHydroquinone Chem. Phys. Lett. 344229

16. Loring R F, Yan Y J and Mukamel S 1985 Generating Function for Electronic Spectra of Polyatomic Molecules J. Phys. Chem. 89201

17. Heller E J 1981 The Semiclassical Way to Molecular Spectroscopy Acc. Chem. Res. 14368

18. Prasad M D 1988 Timedependent Coupled Cluster Method: A New Approach to the Calculation of Molecular Absorption Spectra J. Chem. Phys. 887005

19. Pollak E and He Y 2001 Theory and Control of Thermal Photoinduced Electron Transfer Reactions in Polyatomic Molecules J. Phys. Chem. B $\mathbf{1 0 5} 6500$

20. Cerezo J, Zuniga J, Requena A, Ferrer J A and Santoro F 2013 Harmonic Models in Cartesian and Internal Coordinates to Simulate the Absorption Spectra of Carotenoids at Finite Temperatures J. Chem. Theory Comput. 94947

21. Lax M 1952 The Franck-Condon Principle and Its Application to Crystals J. Chem. Phys. 201752

22. Kubo R and Thoyajowa Y 1955 Application of the Method of Generating Function to Radiative and NonRadiative Transitions of a Trapped Electron in a Crystal Prog. Theor. Phys. 13160

23. Reddy C S and Prasad M D 2015 Finite Temperature Vibronic Spectra of Harmonic Surfaces: A TimeDependent Coupled Cluster Approach Mol. Phys. 113 3023

24. Sridhar Reddy Ch and Durga Prasad M 2016 A Gaussian Wave Packet Propagation Approach to Vibrationally Resolved Optical Spectra at Non-Zero Temperatures $J$. Phys. Chem. A 1202583

25. Baiardi A, Bloino J and Barone V 2013 General Time Dependent Approach to Vibronic Spectroscopy Including Franck-Condon, Herzberg-Teller, and Duschinsky Effects J. Chem. Theory Comput. 94097

26. Niu Y, Peng Q, Deng C, Gao X and Shuai X 2010 Theory of Excited State Decays and Optical Spectra: Application to Polyatomic Molecules J. Phys. Chem. A 1147817

27. Berger R, Fischer C and Klessinger M 1998 Calculation of the Vibronic Fine Structure in Electronic Spectra at Higher Temperatures. 1. Benzene and Pyrazine J. Phys. Chem. A 1027157

28. Yan Y J and Mukamel S 1987 Molecular Fluorescence and Near Resonance Raman Yield as a Probe for Solvation Dynamics J. Chem. Phys. 866085

29. Hajime T and Mitsuo T 1994 Analysis of the absorption spectrum $\left(1^{1} B_{u} \longleftarrow 1^{1} A_{g}\right)$ and resonance Raman excitation profiles of trans $1,3,5$ hexatriene based on ab initio molecular orbital calculations J. Chem. Phys. 1014496

30. Francesco Z 1995 Franck-Condon Modeling of the Structure of the $1 \mathrm{Ag}-1 \mathrm{Bu}$ Electronic Transition of .alpha.,.omega. Diphenylpolyenes J. Am. Chem. Soc. 117 1621

31. Jacquemin D, Planchat A, Adamo C and Mennucci B 2012 TD-DFT Assessment of Functionals for Optical 00 Transitions in Solvated Dyes J. Chem. Theory Comput. 82359
32. Charaf-Eddin A, Planchat A, Mennucci B, Adamo C and Jacquemin D 2013 Choosing a Functional for Computing Absorption and Fluorescence Band Shapes with TD-DFT J. Chem. Theory Comput. 92749

33. Dierksen M and Grimme S 2004 The Vibronic Structure of Electronic Absorption Spectra of Large Molecules: A Time-Dependent Density Functional Study on the Influence of "Exact" Hartree-Fock Exchange J. Phys. Chem. A 10810225

34. Dierksen M and Grimme S 2004 Density Functional Calculations of the Vibronic Structure of Electronic Absorption Spectra J. Chem. Phys. 1203544

35. Muniz-Miranda F, Pedone A, Battisteli G, Montali M, Bloino J and Barone V 2015 Benchmarking TD-DFt against Vibrationally Resolved Absorption Spectra at Room Temperature: 7-Aminocoumarins as Test Cases J. Chem. Theory Comput. 115371

36. Leopoid D G, Vaida V and Granville M F 1984 Direct Absorption Spectroscopy of Jet-Cooled Polyenes. The $11 B+u \leftarrow 11 A-g$ transition of trans-1,3,5,7Octatetraene J. Chem. Phys. 814210

37. Gavin R M, Weisman C, McVey J K and Rice S A 1978 Spectroscopic properties of polyenes. III. 1,3,5,7Octatetraene J. Chem. Phys. 68522

38. Fielding P E and Mackay A G 1964 Vapour Phase Spectrum and Enthalpy of Sublimation of Naphthacene Aust. J. Chem. 171288

39. Heller E J 1975 Time-Dependent Approach to Semiclassical Dynamics J. Chem. Phys. 621544

40. Silva A F, Soares D X, Faria S H D M and Bruns R E 2012 Basis set selection for the calculation of the IR fundamental intensities for $1,1-\mathrm{C}_{2} \mathrm{H}_{2} \mathrm{~F}_{2}$ and $\mathrm{F}_{2} \mathrm{CO} \mathrm{J}$. Mol. Struct. 100949

41. Frisch M J, Trucks G W, Schlegel H B, Scuseria G E, Robb M A, Cheeseman J R, Scalmani G, Barone V, Mennucci B, Petersson G A, Nakatsuji H, Caricato M, Li X, Hratchian H P, Izmaylov A F, Bloino J, Zheng G, Sonnenberg J L, Hada M, Ehara M, Toyota K, Fukuda R, Hasegawa J, Ishida M, Nakajima T, Honda Y, Kitao O, Nakai H, Vreven T, Montgomery J A, Peralta Jr J E, Ogliaro F, Bearpark M, Heyd J J, Brothers E, Kudin K N, Staroverov V N, Keith T, Kobayashi R, Normand J, Raghavachari K, Rendell A, Burant J C, Iyengar S S, Tomas i J, Cossi M, Rega N, Millam J M, Klene M, Knox J E, Crossi J B, Bakken V, Adamo C, Jaramillo J, Gomperts R, Stratmann R E, Yazyev O, Austin A J, Cammi R, Pomelli C, Ochterski J W, Martin R L, Morokuma K, Zakrzewski V G, Voth G A, Salvador P, Dannenberg J J, Dapprich S, Daniels A D, Farkas O, Foresman J B, Ortiz J V, Cioslowski J and Fox D J 2009 Gaussian 09 , Revision C.01; Gaussian Inc., Wallingford CT

42. Avila F J, Improta R and Santoro F 2012 Comparison of Vertical and Adiabatic Harmonic Approaches for the Calculation of the Vibrational Structure of Electronic Spectra Phys. Chem. Chem. Phys. 1413549

43. Parac M and Grimme S 2003 A TDDFT Study of the Lowest Excitation Energies of Polycyclic Aromatic Hydrocarbons Chem. Phys. 29211 